\title{
Optimal electronic doping in $p$-wave superconductors
}

\author{
B. Millán ${ }^{a}$, I. J. Hernández-Hernández ${ }^{b}$, L. A. Pérez ${ }^{c}$, and J. Samuel Millán ${ }^{d, *}$ \\ ${ }^{a}$ Instituto de Investigaciones en Materiales, Universidad Nacional Autónoma de México, \\ Apartado Postal 70-360, 04510, Ciudad de México, México. \\ ${ }^{b}$ Instituto Nacional de Investigaciones Nucleares, \\ Carretera México-Toluca km. 36.5, La Marquesa, 52750, Ocoyoacac, Estado de México, México. \\ ${ }^{c}$ Instituto de Física, Universidad Nacional Autónoma de México, \\ Apartado Postal 20-360, 04510, Ciudad de México, México. \\ ${ }^{d}$ Facultad de Ingeniería, Universidad Autónoma del Carmen, \\ 24180, Cd. del Carmen, Campeche, México. \\ *e-mail: smillan@pampano.unacar.mx
}

Received 15 January 2021; accepted 9 April 2021

Recently, within a generalized Hubbard model which includes correlated nearest $(\Delta t)$ and next-nearest hopping interactions $\left(\Delta t_{3}\right)$, a comparative study between $d$ - and $s^{*}$ - wave superconducting ground states on a square lattice was performed. It was found that the critical temperature of transition $T_{c}(n)$, as a function of the electron concentration $n$, reaches a maximum $\left(T_{c-\text { max }}\right)$ at a given optimal doping $\left(n_{o p}\right)$ for each value of the ratio $t^{\prime} / t$, where $t$ and $t^{\prime}$ are the tight-binding nearest and next-nearest hopping parameter of a square lattice, respectively. From all values obtained for $T_{c-\max }\left(t^{\prime} / t, n_{o p}\right)$ a global minimum one was encountered for both symmetries. Likewise, in the same space, a minimal ground state energy $E_{g}$ was also obtained. For $d$-wave channel both minima are localized around the same optimal doping. However, for $s^{*}$ symmetry, the two minima are located at different electron concentrations. In this work, we additionally study how the $p$-wave ground-state energy and the critical temperature depend on the hoppings parameters and the electron concentration. The results show that for $p$-wave, minimum global values of $T_{c-\max }$ and $E_{g}$ in the $\left(t^{\prime} / t, n_{o p}\right)$ space do exist too, and are found around half filling but, as occurs for $s^{*}$ - wave, the minimum of $T_{c-\text { max }}$ does not occur at the same point as $E_{g}$. Moreover, we present a ground-state phase diagram in the space $\left(t^{\prime} / t, n_{o p}\right)$ where it is possible to find zones of coexistence and competition between the $s^{*}$-, $p$ - and $d$-wave symmetries. Also, an analysis of the shape of the Fermi surface and the single-particle energy, as functions of the wave vector of an electron in the Cooper pair, has been done for different regions of the mentioned space.

Keywords: Theories and models of superconducting state; pairing symmetries (other than s-wave); pseudogap regime.

PACS: 74.20.-z; 74.20.Rp; 74.72.Kf

DOI: https://doi.org/10.31349/RevMexFis.67.061601

\section{Introduction}

The possibility of realization of anisotropic superconductivity with triplet pairing has been subject of interest since the 1970s, when the discovery of superfluity was found in the fermionic isotope ${ }^{3} \mathrm{He}$, with $p$-wave Cooper pairs of atoms and a critical transition temperature of about $1 \mathrm{mK}$ [1]. In 1994, Y. Maeno [2] reported that strontium ruthenate $\left(\mathrm{Sr}_{2} \mathrm{RuO}_{4}\right)$, with a critical temperature of $1.5 \mathrm{~K}$, could possess a superconducting gap with $p$-wave symmetry. In this system, the critical temperature of the superconducting state cannot be tuned by changing the electron density or concentration as in cuprate superconductors such as $\mathrm{La}_{2-x} \mathrm{Sr}_{x} \mathrm{CuO}_{4}$ [3], where an optimal doping of holes $x_{o p} \approx 0.15$ from half filling leads to a maximum critical temperature $T_{c-\max } \approx$ $40 \mathrm{~K}$. Nevertheless, $\mathrm{Sr}_{2} \mathrm{RuO}_{4}(\mathrm{SRO})$ and $\mathrm{La}_{2-x} \mathrm{Sr}_{x} \mathrm{CuO}_{4}$ (LSCO) have similar tetragonal crystal structures but, in the former, the planes where the particles flow without any resistance are $\mathrm{RuO}_{2}$, whereas in the latter are $\mathrm{CuO}_{2}$ [2]. Although $\mathrm{Ru}$ atoms may play an analogous role for electronic pairing as $\mathrm{Cu}$ in LSCO, these systems SRO and LSCO have very distinct superconducting properties; in particular, the former could have $p$-wave Cooper pairs and the latter has a $d$-wave superconducting gap. It has been suggested that in the underdoping regime, for $d$-wave superconductors, the antiferromagnetic state could be a precursor of the superconducting state [4], whereas the ferromagnetic state would be appropriate for $p$-wave superconductors, as possibly occurs in the family of superconductors compounds with uranium (U), such as $\mathrm{UTe}_{2}, \mathrm{UGe}_{2}, \mathrm{URhGe}_{2}$ and UCoGe [5], with critical temperatures around $1.5 \mathrm{~K}$. A model which accounts for both $p$ - and $d$-wave superconductivity, within the same theoretical framework, is the generalized Hubbard model (GHM) [68], where nearest and next-nearest-neighbor correlated hoppings (bond-charge interactions) can lead to the formation of Cooper pairs with different symmetries. For SRO an infinitesimal distortion of the $\mathrm{RuO}_{2}$ square lattice is also considered. This distortion leads to an asymmetry between the second-neighbor correlated hoppings along the two diagonals of the former square lattice, which give rise to two $p$-wave superconducting states of different energies [6]. In previous works, we have studied the dependence of the p-wave 
ground-state critical temperature and $p$-wave gap amplitude on the electron density $(n)$ and the electron-electron interaction parameters within the generalized Hubbard model, maintaining the ratio between first- $(t)$ and second-neighbor $\left(t^{\prime}\right)$ single-electron hoppings constant $[6,7,13]$. Also, we have studied the temperature dependence of the $p$-wave gap amplitude for a fixed set of Hamiltonian parameters which allows the calculation of the temperature behavior of the electronic specific heat, which has shown a good agreement with that experimentally obtained for SRO [13]. However, in a square lattice the ratio $t^{\prime} / t$ determines the energy at which the van Hove singularities occur, that in turn affect the optimal electron density where the maximum $T_{c}(n)$ is attained for a given set of electron-electron interaction parameters. Hence, in this work, in a similar manner as was recently done for $d$ - and $s^{*}$-wave superconductors $[9,10]$, the superconducting critical temperature $\left(T_{c}\right)$ versus the electron density $(n)$ and the ratio $t^{\prime} / t$ is calculated for a set of electron-electron interaction parameters. In particular, for the optimal electron density $\left(n_{o p}\right)$, i.e., that $n$ where the critical temperature attains its maximum $T_{c \text {, max }}$ with the other Hamiltonian parameters fixed, the corresponding ground state energy was determined for different values of $t^{\prime}$. For a given set of electron-electron interaction parameters, the results show that the largest $T_{c, \max }$ occurs at low electron densities, and the minimum of $T_{c, \max }$ can be encountered close to half filling $(n=1)$. Moreover, there could be a competition between $s^{*}-, d$ - and p-wave superconducting states around half filling. Finally, this study suggest that the values of the optimal doping $\left(n_{o p}\right)$ and second-neighbor hopping $\left(t^{\prime}\right)$ that lead to a critical temperature $T_{c} \approx 1.5$ $\mathrm{K}$, which matches with that of the SRO, are found close to have filling $\left(n_{o p} \sim 1\right)$ and for $t^{\prime}<0$, in agreement with first-principles studies performed for SRO [11]. Finally, an analysis of the corresponding Fermi surfaces and the singleparticle excitation energy were performed.

\section{The model}

We start from a single-band generalized Hubbard model onsite $(U)$ and nearest-neighbor $(V)$ Coulombic interactions, first- $(t)$ and second-neighbor $\left(t^{\prime}\right)$ hopping parameters, and first- $(\Delta t)$ and second-neighbor $\left(\Delta t_{3}\right)$ correlated hopping interactions $[6,7]$

$$
\begin{aligned}
H & =t \sum_{<i, j>, \sigma} c_{i \sigma}^{\dagger} c_{j \sigma}+\sum_{\ll i, j \gg, \sigma}\left(t^{\prime}+\chi_{i j} \delta\right) c_{i \sigma}^{\dagger} c_{j \sigma} \\
& +U \sum_{i} c_{i \uparrow} c_{i \downarrow}+\frac{V}{2} \sum_{<i, j>, \sigma} n_{i} n_{j} \\
& +\Delta t \sum_{<i, j>, \sigma} c_{i \sigma}^{\dagger} c_{j \sigma}\left(n_{i,-\sigma}+n_{j,-\sigma}\right) \\
& +\sum_{\substack{<i, l><j, l>\\
\ll i, j \gg, \sigma}}\left(\Delta t_{3}+\chi_{i j} \delta_{3}\right) c_{i \sigma}^{\dagger} c_{j \sigma} n_{l},
\end{aligned}
$$

where $n_{i}=n_{i, \uparrow}+n_{i, \downarrow}, n_{i, \sigma}=c_{i, \sigma}^{\dagger} c_{i, \sigma}$, and $c_{i \sigma}^{\dagger}\left(c_{i \sigma}\right)$, is the creation (annihilation) operator with spin $\sigma=\downarrow$ or $\uparrow$ at site $i .\langle i, j\rangle$ and $\ll i, j \gg$ denote nearest- and the nextnearest neighbour sites, respectively. In the case of SRO, a surface distortion of the $\mathrm{RuO}_{2}$ square lattice has been observed by using X-ray diffraction [12]. Hence, we considered an infinitesimal distortion of the square lattice that leads to an asymmetry between the second-neighbor hopping $\left(t^{\prime}\right)$ and correlated hopping $\left(\Delta t_{3}\right)$ along the two square diagonals. The former undisturbed second-neighbour hopping $\left(t^{\prime}\right)$ and second-neighbor correlated hopping $\left(\Delta t_{3}\right)$ of a non-distorted square lattice respectively become $t^{\prime}+\chi_{i j} \delta$ and $\Delta t_{3}+\chi_{i j} \delta_{3}$, where $\chi_{i j}=+1$ if second-neighbour sites $i$ and $j$ are along the $\hat{\mathbf{x}}-\hat{\mathbf{y}}$ direction, and $\chi_{i j}=-1$ if they are along the $\hat{\mathbf{x}}+\hat{\mathbf{y}} \mathrm{di}-$ rection. Here $\delta$ and $\delta_{3}$ characterize the degree of asymmetry between the mentioned hoppings along the two square diagonals. The expressions for the model parameters are given in Table I in terms of Wannier functions $\left[\varphi\left(\mathbf{r}-\mathbf{R}_{i}\right)\right]$ centred at lattice site $\mathbf{R}_{i}$.

The real-space Hamiltonian (Eq. (1)) can be transformed into reciprocal space by performing a Fourier transform of the electron operators $c_{k, \sigma}^{\dagger}=\left(1 / N_{s}\right) \sum_{j} \exp \left(i \mathbf{k} \cdot \mathbf{R}_{j}\right) c_{j \sigma}^{\dagger}$. After a mean-field Hartree decoupling, the reciprocal-space Hamiltonian can be written as:

$$
\begin{aligned}
\bar{H} & =\sum_{\mathbf{k}, \sigma} \varepsilon_{M F}(\mathbf{k}) c_{k, \sigma}^{\dagger} c_{\mathbf{k}, \sigma}^{\dagger} \\
& +\frac{1}{N_{s}} \sum_{\mathbf{k}, \mathbf{k}^{\prime}, \mathbf{q}, \sigma} W_{\mathbf{k}, \mathbf{k}^{\prime}, \mathbf{q}} c_{\mathbf{k}+\mathbf{q}, \sigma}^{\dagger} c_{-\mathbf{k}+\mathbf{q}, \sigma}^{\dagger} c_{-\mathbf{k}^{\prime}+\mathbf{q}, \sigma} c_{\mathbf{k}}{ }^{\prime}+\mathbf{q}, \sigma
\end{aligned}
$$

TABLE I. Expressions for the Hubbard model parameters.

$$
\begin{aligned}
& \text { Single-particle parameters } \\
& t_{i, j}=\int d^{3} \mathbf{r} \varphi^{*}\left(\mathbf{r}-\mathbf{R}_{i}\right)\left\lfloor-\frac{\hbar^{2} \nabla^{2}}{2 m}+u(\mathbf{r})\right\rfloor \varphi\left(\mathbf{r}-\mathbf{R}_{i}\right) ; u(\mathbf{r}) \text { is the lattice periodic potential } \\
& t=t_{i, j} \text { for }\langle i, j> \\
& t^{\prime}=t_{i, j} \text { for } \ll i, j \gg
\end{aligned}
$$

Electron-electron interaction parameters

$U_{i j}^{k l}=\int d^{3} \mathbf{r} d^{3} \mathbf{r}^{\prime} \varphi^{*}\left(\mathbf{r}-\mathbf{R}_{j}\right) \varphi^{*}\left(\mathbf{r}^{\prime}-\mathbf{R}_{j}\right) v\left(\mathbf{r}-\mathbf{r}^{\prime}\right) \varphi\left(\mathbf{r}-\mathbf{R}_{k}\right) \varphi\left(\mathbf{r}^{\prime}-\mathbf{R}_{l}\right) ;$

$v\left(\mathbf{r}-\mathbf{r}^{\prime}\right)$ is the interaction potential between two electrons in the lattice

$U=U_{i i}^{i i} ; \Delta t=U_{i i}^{i j}$ with $\left\langle i, j>; \Delta t_{3}=U_{l j}^{i l}\right.$ with $\langle i, l>,\langle j, l>$ and $\ll i, j \gg$ 
where the Fourier transform of the electron-electron interaction for electrons with the same spin component is given by:

$$
\begin{aligned}
W_{\mathbf{k}, \mathbf{k}^{\prime}, \mathbf{q}} & =\frac{V}{2} \beta\left(\mathbf{k}-\mathbf{k}^{\prime}\right)+\Delta t_{3}^{+} \gamma\left(\mathbf{k}+\mathbf{q}, \mathbf{k}^{\prime}+\mathbf{q}\right) \\
& +\Delta t_{3}^{-} \zeta\left(\mathbf{k}+\mathbf{q},-\mathbf{k}^{\prime}+\mathbf{q}\right)
\end{aligned}
$$

with

$$
\begin{aligned}
\beta(\mathbf{k}) & =2\left(\cos \left[k_{x} a\right]+\cos \left[k_{y} a\right]\right), \\
\gamma\left(\mathbf{k}, \mathbf{k}^{\prime}\right) & =2 \cos \left(a\left[k_{x}+k_{y}^{\prime}\right]\right)+2 \cos \left(a\left[k_{x}^{\prime}+k_{y}\right]\right), \\
\zeta\left(\mathbf{k}, \mathbf{k}^{\prime}\right) & =2 \cos \left(a\left[k_{x}-k_{y}^{\prime}\right]\right)+2 \cos \left(a\left[k_{x}^{\prime}-k_{y}\right]\right),
\end{aligned}
$$

where $a$ is the lattice parameter and $\Delta t_{3}^{ \pm}=\Delta t_{3} \pm \delta_{3}$. Notice that the real-space term $\Delta t_{3}+\chi_{i j} \delta_{3}$ in Eq. (1) leads, after the Fourier transform, to $\Delta t_{3}^{+}$and $\Delta t_{3}^{-}$depending on the value of $\chi_{i j}( \pm 1)$. Also, $\varepsilon_{M F}(\mathbf{k})$ is the mean-field dispersion relation and $N_{s}$ is the total number of lattice sites. In this case, $\varepsilon_{M F}(\mathbf{k})$ is given by:

$$
\begin{aligned}
\varepsilon_{M F}(\mathbf{k}) & =E_{M F}+2 t_{M F}\left(\cos \left[k_{x} a\right]+\cos \left[k_{y} a\right]\right) \\
& +2 t_{M F,+}^{\prime} \cos \left(a\left[k_{x}+k_{y}\right]\right) \\
& +2 t_{M F,-}^{\prime} \cos \left(a\left[k_{x}-k_{y}\right]\right),
\end{aligned}
$$

with

$$
\begin{aligned}
E_{M F} & =\left(\frac{U}{2}+4 V\right) n, \\
t_{M F} & =t+n \Delta t, \\
t_{M F, \pm}^{\prime} & =t_{ \pm}^{\prime}+2 n \Delta t_{3}^{ \pm}=t^{\prime} \pm \delta+2 n\left(\Delta t_{3} \pm \delta_{3}\right),
\end{aligned}
$$

where $n$ is the electron density and $t_{ \pm}^{\prime}=t^{\prime} \pm \delta$. Notice that the mean-field single-particle hoppings $t_{M F}$ and $t_{M F, \pm}^{\prime}$, depend on the electron density $(n)$ and correlated hoppings $\left(\Delta t, \Delta t_{3}\right)$, in particular on the distorted values of the secondneighbor correlated hopping $\left(\Delta t_{3}^{ \pm}=\Delta t_{3} \pm \delta_{3}\right)$.

In general, the BCS coupled integral equations can be written as [6,7]:

$$
\Delta(\mathbf{k})=-\frac{1}{2 N_{S}} \sum_{\mathbf{k}^{\prime}} \frac{W_{\mathbf{k}, \mathbf{k}^{\prime}, 0} \Delta\left(\mathbf{k}^{\prime}\right)}{E\left(\mathbf{k}^{\prime}\right)} \tanh \left(\frac{E\left(\mathbf{k}^{\prime}\right)}{2 k_{B} T}\right),
$$

and

$$
n-1=-\frac{1}{2 N_{S}} \sum_{\mathbf{k}^{\prime}} \frac{\varepsilon_{M F}\left(\mathbf{k}^{\prime}\right)-\mu}{E\left(\mathbf{k}^{\prime}\right)} \tanh \left(\frac{E\left(\mathbf{k}^{\prime}\right)}{2 k_{B} T}\right),
$$

whose solution gives the chemical potential $(\mu)$ and the amplitude of the superconducting gap for given $n$ and $T$. For the particular case of an interaction $W_{\mathbf{k}, \mathbf{k}, \mathbf{q}}$ given by Eq. (3), Eqs. (11) and (12) admit a solution for the superconducting gap $(\Delta(\mathbf{k}))$ of the form:

$$
\Delta_{p}(\mathbf{k})=\Delta_{p}\left(\sin \left[k_{x} a\right] \pm \sin \left[k_{y} a\right]\right),
$$

known as $p$-wave superconducting gap, where $\Delta_{p}$ is the temperature-dependent superconducting gap amplitude and the sign \pm indicates the direction of the real-space distortion. It is worth mentioning that, in general, the gap of a superconducting state formed by triplet Cooper pairs could have another symmetry different from $p$-wave, such as $f$-wave symmetry, and the possible symmetries depend on the form of the electron-electron interaction potential $W_{\mathbf{k}, \mathbf{k}, \mathbf{q}}$.

By substituting (13) in Eqs. (11) and (12) we obtain:

$$
\begin{aligned}
1 & =-\frac{\left(V \mp 4 \delta_{3}\right) a^{2}}{4 \pi^{2}} \iint_{1 B Z} \frac{\left(\sin \left[k_{x} a\right] \pm \sin \left[k_{y} a\right]\right)^{2}}{2 E(\mathbf{k})} \\
& \times \tanh \left(\frac{E(\mathbf{k})}{2 k_{B} T}\right) d k_{x} d k_{y}, \\
1-n & =-\frac{a^{2}}{4 \pi^{2}} \iint_{1 B Z} \frac{\varepsilon_{M F}(\mathbf{k})-\mu}{E(\mathbf{k})} \\
& \times \tanh \left(\frac{E(\mathbf{k})}{2 k_{B} T}\right) d k_{x} d k_{y},
\end{aligned}
$$

where the sums over the first Brillouin zone (1BZ), defined as

$$
\left[\frac{-\pi}{a}, \frac{\pi}{a}\right] \otimes\left[\frac{-\pi}{a}, \frac{\pi}{a}\right]
$$

have been transformed into integrals. Here $E(\mathbf{k})$ is the quasiparticle energy given by:

$$
E(\mathbf{k})=\sqrt{\left(\varepsilon_{M F}(\mathbf{k})-\mu\right)^{2}+\Delta_{p}^{2}(\mathbf{k})} .
$$

It is important to mention that the chiral $p$-wave gap given by

$$
\Delta_{\text {chi-p }}(\mathbf{k})=\Delta_{\text {chi }-p}\left(\sin \left[k_{x} a\right] \pm i \sin \left[k_{y} a\right]\right),
$$

also satisfies Eqs. (11) and (12) with $W_{\mathbf{k}, \mathbf{k}, \mathbf{q}}$ given by Eq. (3), where the second term in the square root of Eq. (16) is

$$
\begin{aligned}
\Delta_{\text {chi }-p}(\mathbf{k}) & \Delta_{\text {chi-p }}^{*}(\mathbf{k})=\Delta_{\text {chi-p }}^{2} \\
& \times\left(\sin ^{2}\left[k_{x} a\right]+\sin ^{2}\left[k_{y} a\right]\right),
\end{aligned}
$$

instead of

$$
\Delta_{p}^{2}(\mathbf{k})=\Delta_{p}^{2}\left(\sin \left[k_{x} a\right] \pm \sin \left[k_{y} a\right]\right)^{2} .
$$

However, in this work, the chiral case will not be considered because it does not seem to successfully apply to SRO [13].

The critical temperature $T_{c}$ can be determined from Eqs. (14) and (15) considering that $\Delta_{p}\left(T=T_{c}\right)=0$. In 
this case, these equations transform into:

$$
\begin{aligned}
1 & =-\frac{\left(V \pm 4 \delta_{3}\right) a^{2}}{4 \pi^{2}} \iint_{1 B Z} \frac{\left(\sin \left[k_{x} a\right] \pm \sin \left[k_{y} a\right]\right)^{2}}{2\left|\varepsilon_{M F}(\mathbf{k})-\mu\right|} \\
& \times \tanh \left(\frac{\mid \epsilon_{M F}(\mathbf{k}-\mu \mid}{2 k_{B} T_{c}}\right) d k_{x} d k_{y}, \\
n-1 & =-\frac{a^{2}}{4 \pi^{2}} \iint_{1 B Z} \frac{\varepsilon_{M F}(\mathbf{k})-\mu}{\left|\varepsilon_{M F}(\mathbf{k})-\mu\right|} \\
& \times \tanh \left(\frac{\left|\varepsilon_{M F}(\mathbf{k})-\mu\right|}{2 k_{B} T_{c}}\right) d k_{x} d k_{y} .
\end{aligned}
$$

Here, for given Hamiltonian interaction parameters and $n$, the two-coupled equations determine the values of $T_{c}$ and $\mu$. Moreover, the ground state energy $\left(E_{g}\right)$ per site is given by [14]:

$$
\begin{aligned}
E_{g} & =\frac{1}{N_{s}} \sum_{\mathbf{k}}\left(\varepsilon_{M F}(\mathbf{k})-\mu\right)-\frac{1}{N_{s}} \sum_{\mathbf{k}} E(\mathbf{k}) \\
& +\frac{1}{N_{s}} \sum_{\mathbf{k}} \frac{\Delta^{2}(\mathbf{k})}{2 E(\mathbf{k})}+\mu N-\left(\frac{U}{4}+2 V\right) n^{2},
\end{aligned}
$$

which, in the case of a $p$-symmetry superconducting gap Eq. (13) can be simplified to:

$$
\begin{aligned}
E_{g} & =\frac{1}{N_{s}} \sum_{\mathbf{k}}\left[\varepsilon_{M F}(\mathbf{k})-E(\mathbf{k})\right]+\frac{\Delta_{p}^{2}}{4 \delta_{3}-V} \\
& +(n-1) \mu-\left(\frac{U}{4}+2 V\right) n^{2} .
\end{aligned}
$$

It is important to mention that for $p$-wave superconductors, $U$ plays a similar role in the superconducting gap equation as for $d$-wave ones, i.e., it does not affect the shape of $\varepsilon_{M F}$, and only modifies the electron self-energy $\left(E_{M F}\right)$ shifting the numerical value of the superconducting chemical potential $(\mu)$ without changing the superconducting critical temperature. Therefore $U$ can be taken equal to zero. Moreover, to obtain a solution for the superconducting gap equation [Eq. (7)], $\left(V-4 \delta_{3}\right)$ should be negative and therefore the condition $V<4 \delta_{3}$ must to be satisfied. In order to keep a minimum set of parameters but considering that a p-wave superconducting gap can be generated from $\delta_{3}, V$ will be set to zero too. It is worth mentioning that models which consider a negative $V$ can lead to triplet pairing in frustrated lattices [15], however, this term would also lead to a phase separated state, where electrons double occupy sites over a macroscopic region of the lattice, which would strongly compete with the superconducting state [16].

\section{Results}

Figure 1a) shows the critical temperature $T_{c}$ of $p$-wave superconducting states as a function of the electron concentration $n$ and the second-neighbor hopping parameter $t^{\prime}$ for $\Delta t=0.5|t|$,
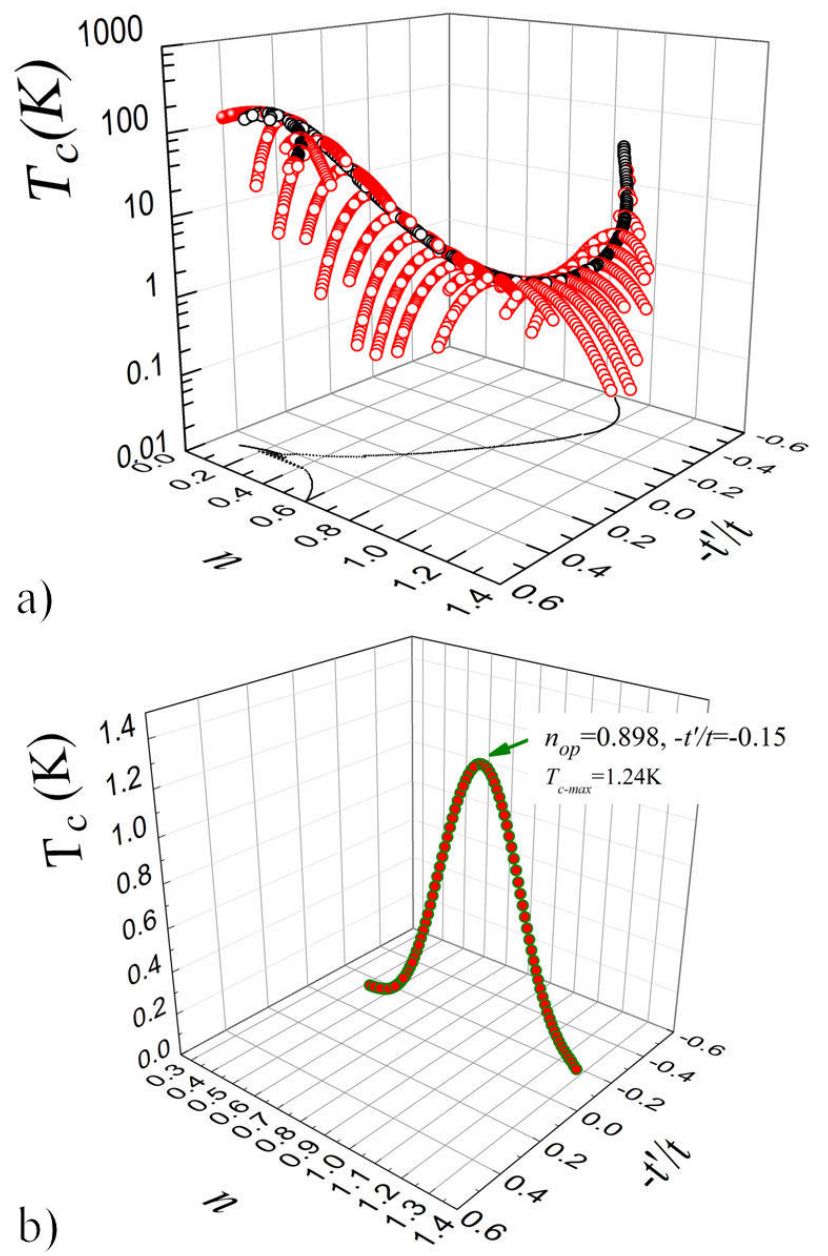

FIGURE 1. a) Critical temperature $\left(T_{c}\right)$ versus electronic density $(n)$ and $t^{\prime} / t$ for $p$-wave superconductors with $U=V=0$, $\Delta t=0.5|t|, \delta=\Delta t_{3}=0.05|t|$, and $\delta_{3}=0.08|t|$ (red circles), with $t=-1 \mathrm{eV}$. The dark gray circles corresponds depicts the maximum $T_{c}$ for each value of $t^{\prime} / t$. b) Critical temperature $\left(T_{c}\right)$ versus electronic density $(n)$ for a $p$-wave superconductor with $t^{\prime} / t=-0.15$ and the same interaction parameters as in Fig. 1a).

$\Delta t_{3}=\delta=0.05|t|$, and $\delta_{3}=0.08|t|$. Likewise, Fig. 1b) shows the critical temperature versus the electronic density ( $n$ ) for a particular value of $-t^{\prime} / t=-0.15$. The value of $n$, where the maximum critical temperature $\left(T_{c-\max }\right)$ is attained for each value of $t^{\prime} / t$ is called the optimal electron density $n_{o p}$.

The $p$-channel maximum critical temperature $\left(T_{c-\max }\right)$ versus the optimal electronic density $\left(n_{o p}\right)$ and $t^{\prime} / t$, for the same interaction parameters $\Delta t$ and $\Delta t_{3}$ can be obtained from Fig. 1a). These curves are shown in Fig. 2a) for different values of $\delta_{3}$. The projections of these curves $T_{c-\max }\left(n_{o p}, t^{\prime} / t\right)$ on the $\left(n_{o p}, t^{\prime} / t\right)$ plane are presented in Fig. 2b). For a given $\delta_{3}$, a global maximum of $T_{c-\max }$ can be found for $t^{\prime}>0$ (solid magenta triangles) within of the intervals $n_{o p-\max } \in[0.08,0.32]$ and $t^{\prime} \in[0.47,0.43]$. The latter interval is very narrow. On the other hand, for a given $\delta_{3}$, minimum values of $T_{c-\max }$ can be found for $t^{\prime}<0$. These 
TABLE II. Maximum and minimum values of $T_{c-\max }$ with the corresponding ground state energies for $p$-wave superconductors with $\Delta t=0.5 \mathrm{eV}, \delta=\Delta t_{3}=0.05 \mathrm{eV}$ and different values of $\delta_{3}$.

\begin{tabular}{ccccccccc}
\hline$\delta_{3}(\mathrm{eV})$ & $n_{o p}$ & $-t^{\prime} / t$ & $\operatorname{Max}\left[T_{c-\max }\right](\mathrm{K})$ & $E_{g}(\mathrm{meV})$ & $n_{o p}$ & $-t^{\prime} / t$ & $\operatorname{Min}\left[T_{c-\max }\right](\mathrm{K})$ & $E_{g}(\mathrm{meV})$ \\
\hline 0.100 & 0.320 & 0.429250 & 285.18 & -554.862 & 0.863 & -0.190 & 6.14 & -1035.20 \\
0.090 & 0.295 & 0.429625 & 228.55 & -515.152 & 0.884 & -0.175 & 3.00 & -1003.648 \\
0.080 & 0.265 & 0.431875 & 177.77 & -467.880 & 0.898 & -0.150 & 1.24 & -973.712 \\
0.070 & 0.225 & 0.437500 & 133.57 & -404.334 & 0.913 & -0.130 & 0.40 & -946.0469 \\
0.060 & 0.185 & 0.445550 & 96.34 & -338.897 & 0.921 & -0.105 & 0.09 & -923.498
\end{tabular}
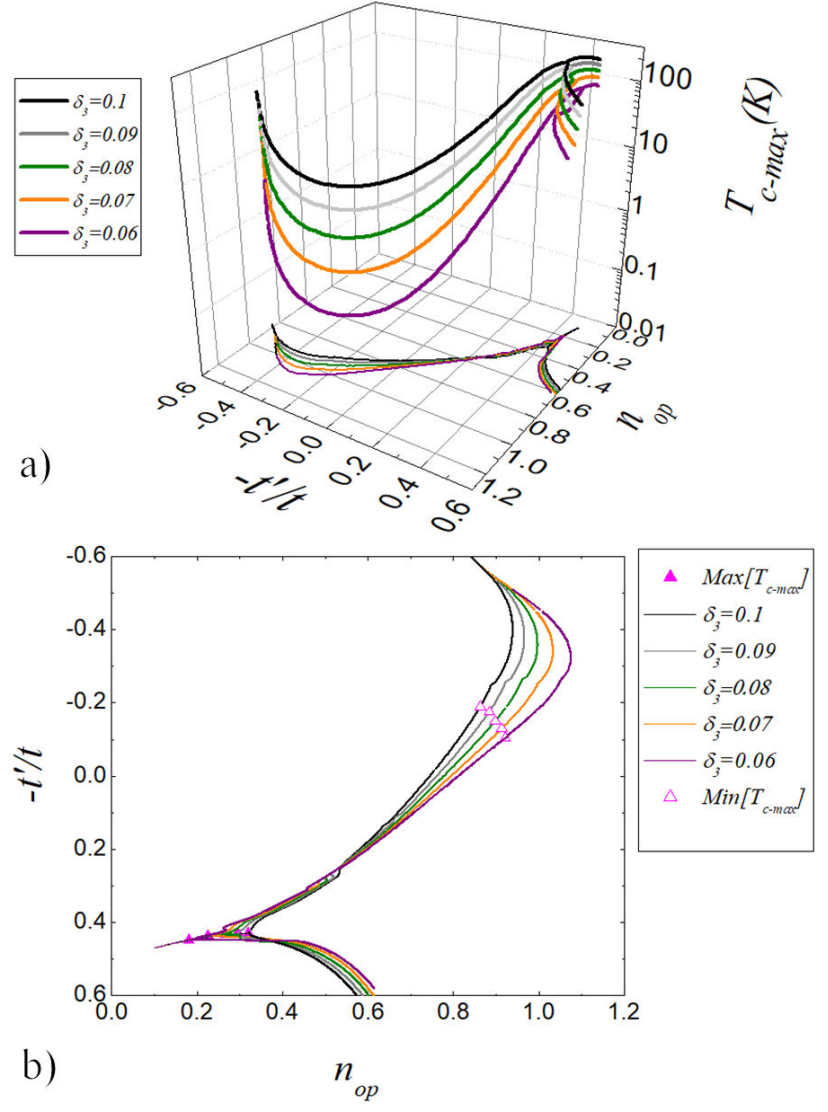

FIGURE 2. a) Maximum superconducting critical temperature $\left(T_{c-\max }\right)$ versus the optimal electronic density $\left(n_{o p}\right)$ and $-t^{\prime}$ for $U=V=0, \Delta t=0.5|t|, \delta=\Delta t_{3}=0.05|t|$, and $\delta_{3} \in$ $[0.06|t|, 0.1|t|]$ (colors lines). b) Projections of the curves depicted in Fig. $2 \mathrm{a})$ on the plane $\left(n_{o p},-t^{\prime} / t\right)$. The magenta solid (open) triangles correspond to maxima (minima) of $T_{c-\max }$.

minima are located within the intervals $n_{o p} \in[0.863,0.921]$ and $t^{\prime} \in[-0.190,-0.105]$ (open magenta triangles). The values of $n_{o p}$ and $t^{\prime}$ where the global maxima and minima of $T_{c-\max }$ occur, for each $\delta_{3}$, are shown in Table II.

It is important to underline that for $t^{\prime}<0, n_{o p}$ is close to half filling and, in this region, $T_{c-\max }$ abruptly diminishes. Moreover, as shown below, in this zone $p$-wave superconducting states compete with extended $s$-wave $\left(s^{*}\right)$ and $d$-wave ones.

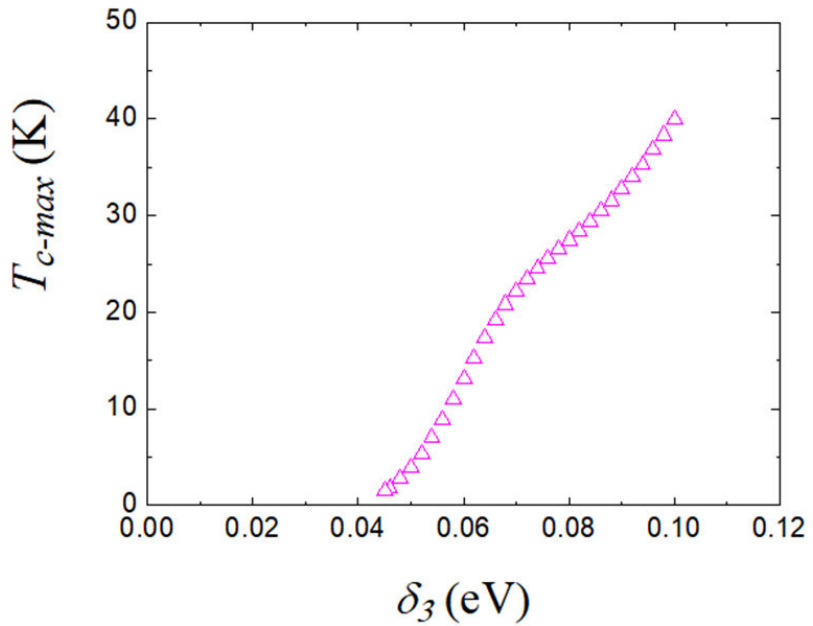

FIGURE 3. Maximum critical temperature $\left(T_{c-\max }\right)$ as a function of $\delta_{3}$ for $\left(n_{o p},-t^{\prime} / t\right)=(0.868,-0.57)$.

It is also worth mentioning that close to $\left(n_{o p},-t^{\prime} / t\right)=$ $(0.868,-0.57), n_{o p}$ is almost the same for all $\delta_{3}$. Figure 3 shows $T_{c-\max }$ versus $\delta_{3}$ at this point.

Moreover, when $\delta_{3}=0.045 \mathrm{eV}$, the maximum critical temperature $\left(T_{c-\max }\right)$ of the state $\left(n_{o p},-t^{\prime} / t\right)=$ $(0.868,-0.57)$ agrees with the critical temperature of SRO, i.e., $T_{c} \approx 1.5 \mathrm{~K}$. Other points that satisfy $T_{c-\max } \approx 1.5 \mathrm{~K}$ with different values of $\delta_{3}$ are summarized in Table III. Two cases correspond to $t^{\prime}>0:\left(n_{o p},-t^{\prime} / t\right)=(0.684,0.115)$ with $\delta_{3}=0.07 \mathrm{eV}$; and $\left(n_{o p},-t^{\prime} / t\right)=(0.586,0.210)$ with $\delta_{3}=0.06 \mathrm{eV}$. The other cases correspond to $t^{\prime}<0$. Taken into account that the distortion of the square lattice is assumed to be very small, it is expected that the parameter $\delta_{3}$ should be very small. Hence, according with the results of this work, the smaller value of $\delta_{3}$ satisfying $T_{c-\max } \approx 1.5 \mathrm{~K}$, corresponds to $\delta_{3}=0.045 \mathrm{eV}$, which is attained at $\left(n_{o p},-t^{\prime} / t\right)=(0.868,-0.57)$. However, it has been estimated, by using first-principles calculations [11], that the first- and second-neighbor hoppings of SRO on the $\mathrm{RuO}_{2}$ planes satisfy $-t^{\prime} / t=-0.3$ and then, the states $\left(n_{o p},-t^{\prime} / t\right)=(0.960,-0.26)$ with $\delta_{3}=0.08$ and $(1.027,-0.385)$ with $\delta_{3}=0.07 \mathrm{eV}$, both of which also have small values of $\delta_{3}$ and optimal electron concentrations (dopings) close to half-filling, are good candidates to be the experimentally observed $p$-wave ground state of SRO. 


\begin{tabular}{ccccccccc}
\hline \hline \multicolumn{7}{c}{ TABLE III. Superconducting physical properties of $\left(n_{o p},-t^{\prime} / t\right) p$-wave states satisfying $T_{c-\max }=1.5 \mathrm{~K}}$. \\
\hline$\delta_{3}(\mathrm{eV})$ & $n_{o p}$ & $-t^{\prime} / t$ & $T_{c-\max }(\mathrm{K})$ & $\Delta_{p-\max }(\mathrm{meV})$ & $\Delta_{0}(\mathrm{meV})$ & $E_{g}(\mathrm{meV})$ & $E_{(}(\mathrm{con})(\mathrm{meV})$ & $E_{F}(\mathrm{meV})$ \\
\hline 0.08 & 0.815 & -0.045 & 1.50 & 0.169 & 0.335 & -981.456 & -0.007 & -322.263 \\
0.08 & 0.960 & -0.260 & 1.50 & 0.174 & 0.302 & -960.071 & -0.214 & 265.916 \\
0.07 & 0.684 & 0.115 & 1.47 & 0.171 & 0.341 & -925.830 & -51.9 & -811.084 \\
0.07 & 1.027 & -0.385 & 1.52 & 0.178 & 0.233 & -924.312 & $6.0 \times 10^{-6}$ & 494.374 \\
0.06 & 0.586 & 0.210 & 1.46 & 0.179 & 0.347 & -849.282 & $-3.7 \times 10^{-6}$ & -1108.611 \\
0.06 & 1.008 & -0.450 & 1.49 & 0.182 & 0.183 & -962.571 & -0.036 & 498.879 \\
0.045 & 0.868 & -0.570 & 1.50 & 0.121 & 0.335 & -1174.628 & -0.0145 & 364.411 \\
\hline
\end{tabular}

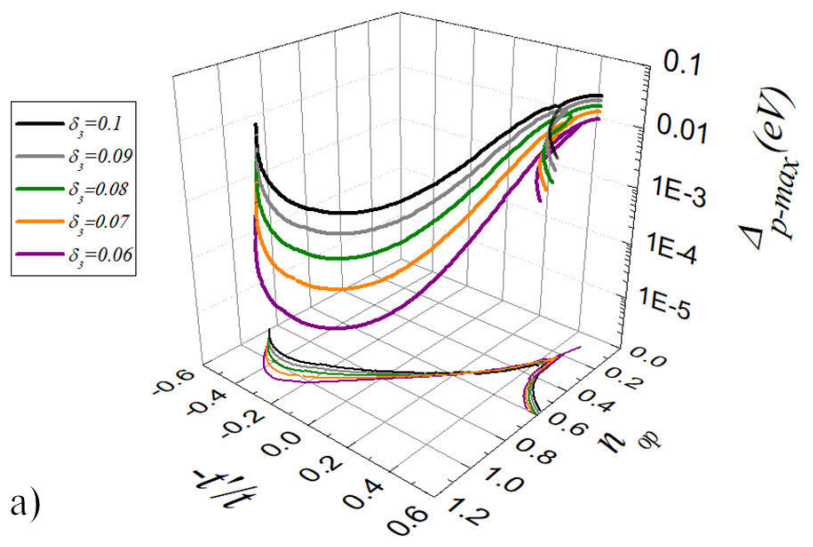

tice that the FS are symmetric with respect to the $\mathbf{k}_{x}+\mathbf{k}_{y}$ diagonal. It is also important to point out that the states $\left(n_{o p}=0.586, t^{\prime}=0.2|t|, \delta_{3}=0.06 \mathrm{eV}\right),\left(n_{o p}=0.684\right.$, $\left.t^{\prime}=0.115|t|, \delta_{3}=0.07 \mathrm{eV}\right),\left(n_{o p}=0.815, t^{\prime}=-0.045|t|\right.$, $\delta_{3}=0.08 \mathrm{eV}$ ) whose single-particle excitation gaps are depicted in Figs. 6a)-6c), $\Delta_{0} \approx 2 \Delta_{p}$ at the antinode. However, for the other cases, the FS is more elongated along the $\mathbf{k}_{x}+\mathbf{k}_{y}$ diagonal, and the relation $\Delta_{0} \approx 2 \Delta_{p}$ is not fulfilled at the antinode. In the case $\left(n_{o p}=0.868, t^{\prime}=-0.570|t|\right.$, $\delta_{3}=0.045 \mathrm{eV}$ ), the FS is disconnected, see Fig. $5 \mathrm{~d}$ ).

Besides $p$-wave superconducting ground states, the first$(\Delta t)$ and second-neighbor $\left(\Delta t_{3}\right)$ correlated hoppings can also induce extended $s$-wave $\left(s^{*}\right)$ and d-wave superconducting states [7], where the respective gaps (order parameters) are given by $\Delta_{s^{*}}(\mathbf{k})=\Delta_{s}+\Delta_{s^{*}}\left[\cos \left(k_{x} a\right)+\cos \left(k_{y} a\right)\right]$ and $\Delta_{d}(\mathbf{k})=\Delta_{d}\left(\cos \left[k_{x} a\right]-\cos \left[k_{y} a\right]\right)$. To compare the behavior of $p$-wave superconducting systems with those with extended $s$ - and $d$-wave superconducting gaps, Fig. 7a) shows those points $\left(n_{o p},-t^{\prime} / t\right)$ where the critical temperature is maximum, for $s^{*}$ - (black line), $p$ - (purple line), and $d$-wave (gray line) superconductors. The depicted $d$ - and $s^{*}$-wave states have $\Delta t=0.5|t|$, and $\Delta t_{3}=0.05|t|[9,10]$, whereas the $p$-wave ones have, in addition, $\delta=0.05 \mathrm{eV}$ and $\delta_{3}=0.06$ $\mathrm{eV}$. In each curve, the red open and solid circles correspond to those states where $T_{c-\max }$, and $E_{g}$, respectively attain their minimum values. Figure $7 \mathrm{~b}$ ) shows the corresponding ground state energies for the same systems as in Fig. 7a). Notice that, at low densities, around the point $\left(n_{o p},-t^{\prime} / t\right)=$ $(0.14,0.455)$, all the curves coincide, and the ordering from lowest to highest value of $E_{g}$ correspond to $s^{*}$-, $p$ - and $d$ wave symmetries. However, for higher densities, the maximum critical temperatures for the different superconducting symmetries are located in different regions, and can be distinguished two crossing points; one around $\left(n_{o p},-t^{\prime} / t\right)=$ $(0.260,0.415)$, where the $s *$ channel curve crosses with the p-channel one, and other at $\left(n_{o p},-t^{\prime} / t\right)=(0.459,0.310)$, where the $d$-channel crosses with the $p$-channel one. Close to these points it is expected that the different possible superconducting symmetries could coexist, if they had similar ground state energies.

Table IV summarizes the superconducting physical properties of the mentioned points of possible coexistence. 


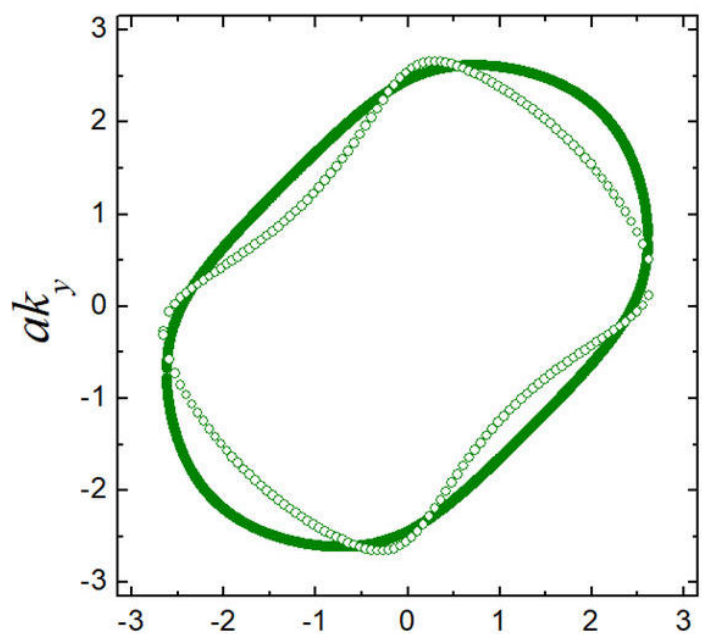

a) $\delta_{3}=0.08 \mathrm{eV}$

- $n=0.960, t^{\prime}=-0.26 \mathrm{eV} \quad a k_{x}$

- $n=0.815, t^{\prime}=-0.045 \mathrm{eV}$
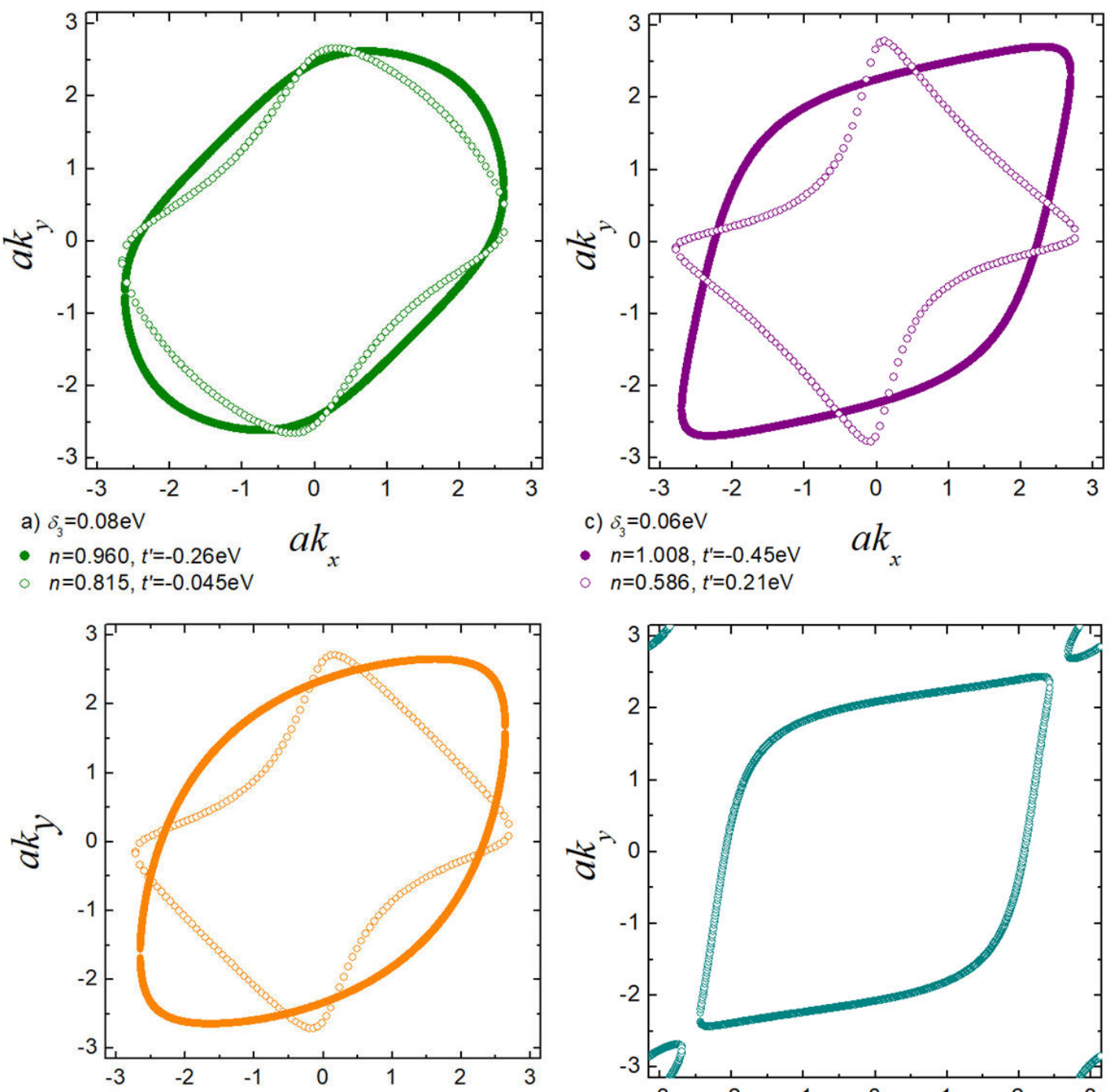

c) $\delta_{3}=0.06 \mathrm{eV}$

- $n=1.008, t^{\prime}=-0.45 \mathrm{eV} \quad a k_{x}$

- $n=0.586, t^{\prime}=0.21 \mathrm{eV}$

b) $\delta_{3}=0.07 \mathrm{eV}$

- $n=1.027, t^{\prime}=-0.385 \mathrm{eV} \quad a k_{x}$

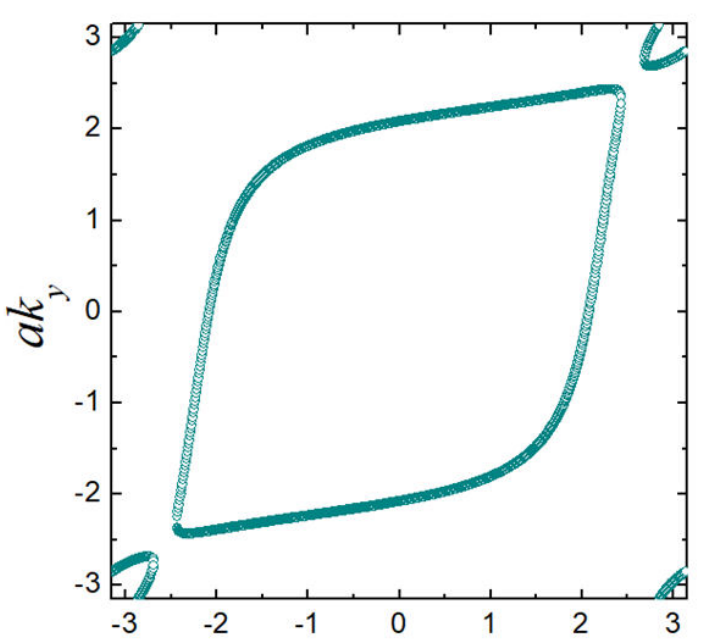

$n=0.684, t^{\prime}=0.115 \mathrm{eV}$

d) $\delta_{3}=0.045 \mathrm{eV}$

○ $n=0.868, t^{\prime}=-0.57 \mathrm{eV} \quad a k_{x}$

FIGURE 5. Fermi surface of $p$-wave superconducting states with a) $\left(n_{o p}=0.815, t^{\prime}=-0.045|t|, \delta_{3}=0.08 \mathrm{eV}\right)$, b) $\left(n_{o p}=0.684\right.$, $\left.\left.t^{\prime}=0.115|t|, \delta_{3}=0.07 \mathrm{eV}\right), \mathrm{c}\right)\left(n_{o p}=0.586, t^{\prime}=0.2|t|, \delta_{3}=0.06 \mathrm{eV}\right)$, and d) $\left(n_{o p}=0.868, t^{\prime}=-0.570|t|, \delta_{3}=0.045 \mathrm{eV}\right)$.

TABLE IV. Superconducting physical properties of $s^{*}, p$ and $d$-wave superconducting states with $\Delta t=0.5 \mathrm{eV}, \Delta t_{3}=0.05 \mathrm{eV}$, $U=2.0 \mathrm{eV}$ and $\delta=\delta_{3}=0$ ( $s^{*}$ - and $d$-wave) or $\delta=0.05 \mathrm{eV}, \delta_{3}=0.06 \mathrm{eV}$ ( $p$-wave).

\begin{tabular}{cccccccc}
\hline Symmetry $\alpha$ & $n_{o p}$ & $-t^{\prime} / t$ & $T_{c}(\mathrm{~K})$ & $E_{g}(\mathrm{meV})$ & $\Delta_{\alpha}(\mathrm{meV})$ & $E_{\text {cond }}(\mathrm{meV})$ & $E_{F}(\mathrm{meV})$ \\
\hline$s^{*}$ & 0.140 & 0.455 & 162.53 & -613.027 & -022.126, & 0.700 & -1707.073 \\
$s$ & & & & & 16.962 & & \\
$\mathrm{p}$ & 0.140 & 0.4572 & 93.87 & -262.703 & 14.559 & 0.245 & -1863.602 \\
$\mathrm{~d}$ & 0.140 & 0.455 & 129.36 & -260.216 & 14.501 & 0.241 & -1844.425 \\
$\mathrm{~s} *$ & 0.260 & 0.414 & 168.50 & -690.708 & -17.926 & 1.839 & -1447.564 \\
$\mathrm{~s}$ & & & & & 13.993 & & \\
$\mathrm{p}$ & 0.260 & 0.415 & 77.8 & -456.167 & 10.753 & 0.152 & -1723.577 \\
$\mathrm{~d}$ & 0.455 & 0.310 & 126.14 & -700.532 & 11.630 & 0.152 & -1410.959 \\
$\mathrm{p}$ & 0.459 & 0.310 & 10.31 & -719.364 & 1.3490 & 0.001 & -1421.150 \\
\hline
\end{tabular}



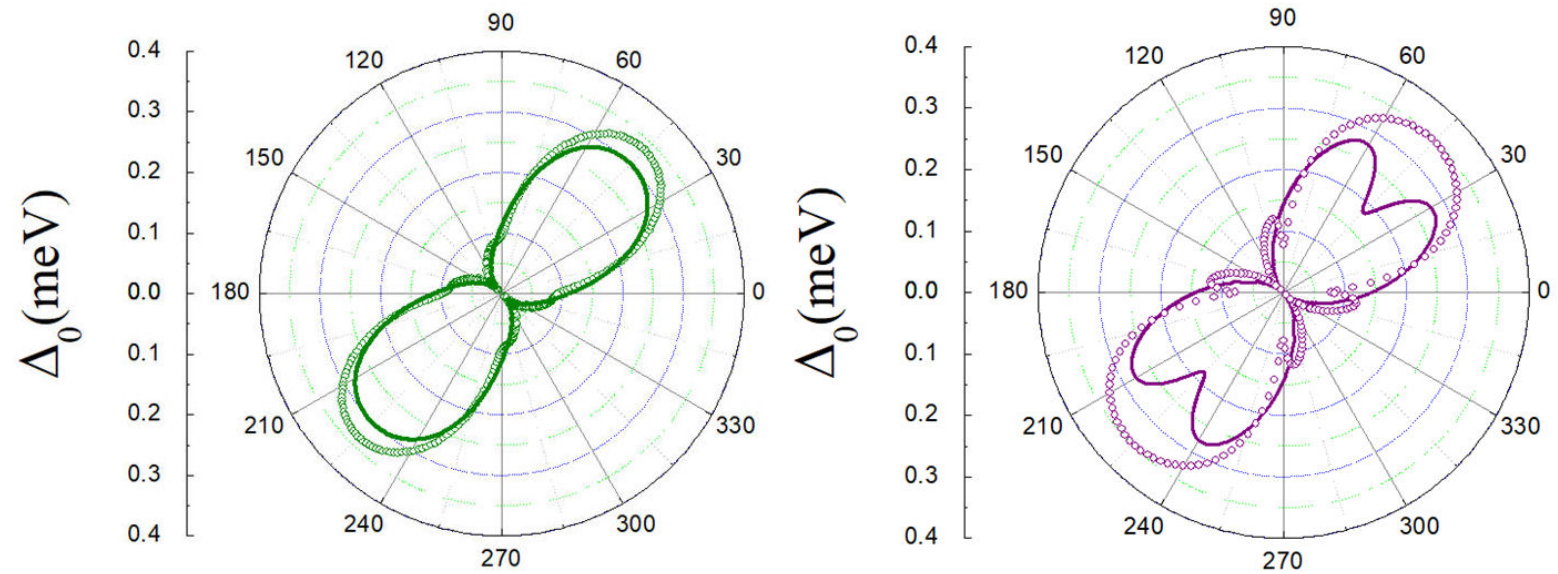

a) $\begin{aligned} \delta_{3} & =0.08 \mathrm{eV} \\ n & =0.960, t^{\prime}=-0.26 \mathrm{eV} \\ n & =0.815, t^{\prime}=-0.045 \mathrm{eV}\end{aligned}$

$$
\text { c) } \begin{aligned}
\delta_{3} & =0.06 \mathrm{eV} \\
n & =1.008, t^{\prime}=-0.45 \mathrm{eV} \\
n & =0.586, t^{\prime}=0.21 \mathrm{eV}
\end{aligned}
$$
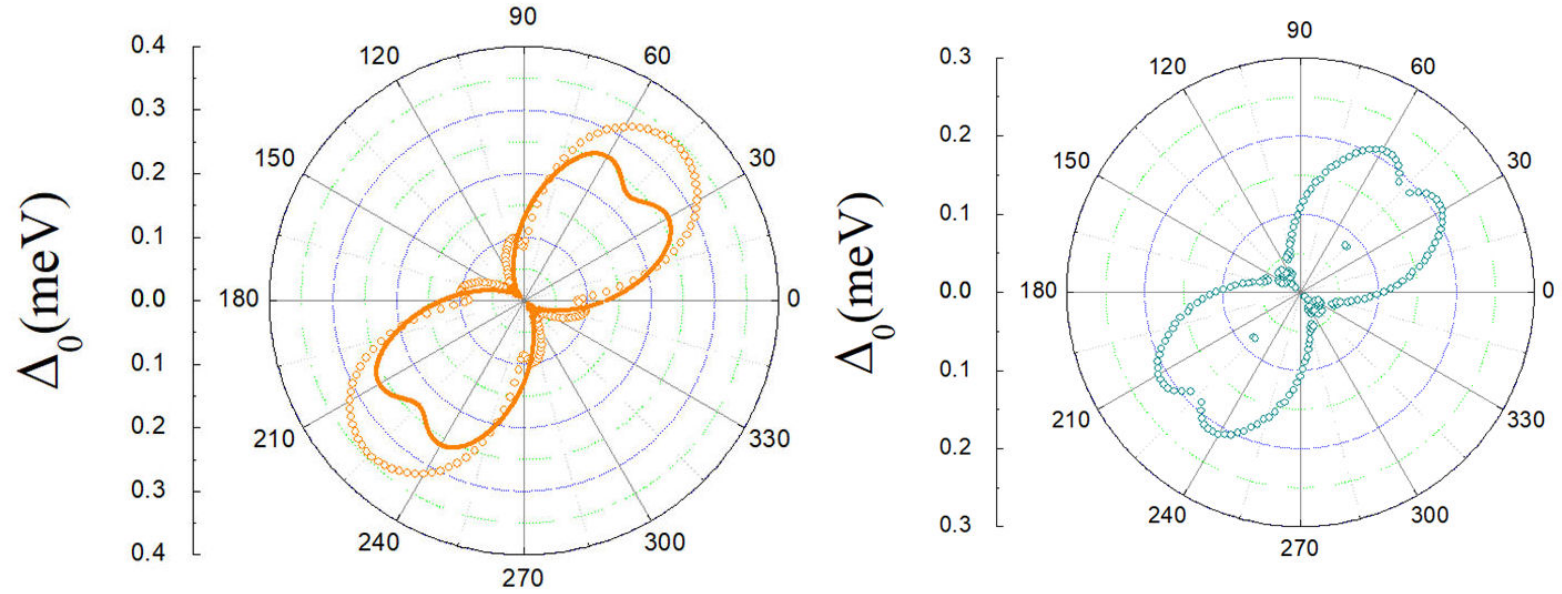

b) $\begin{aligned} \delta_{3} & =0.07 \mathrm{eV} \\ n & =1.027, t^{\prime}=-0.385 \mathrm{eV} \\ n & =0.684, t^{\prime}=0.115 \mathrm{eV}\end{aligned}$

d) $\delta_{3}=0.045 \mathrm{eV}$

$n=0.868, t^{\prime}=-0.57 \mathrm{eV}$

FIGURE 6. Single-particle excitation energies $\left(\Delta_{0}\right)$ for the same systems as Figs. 5a)-d), respectively.
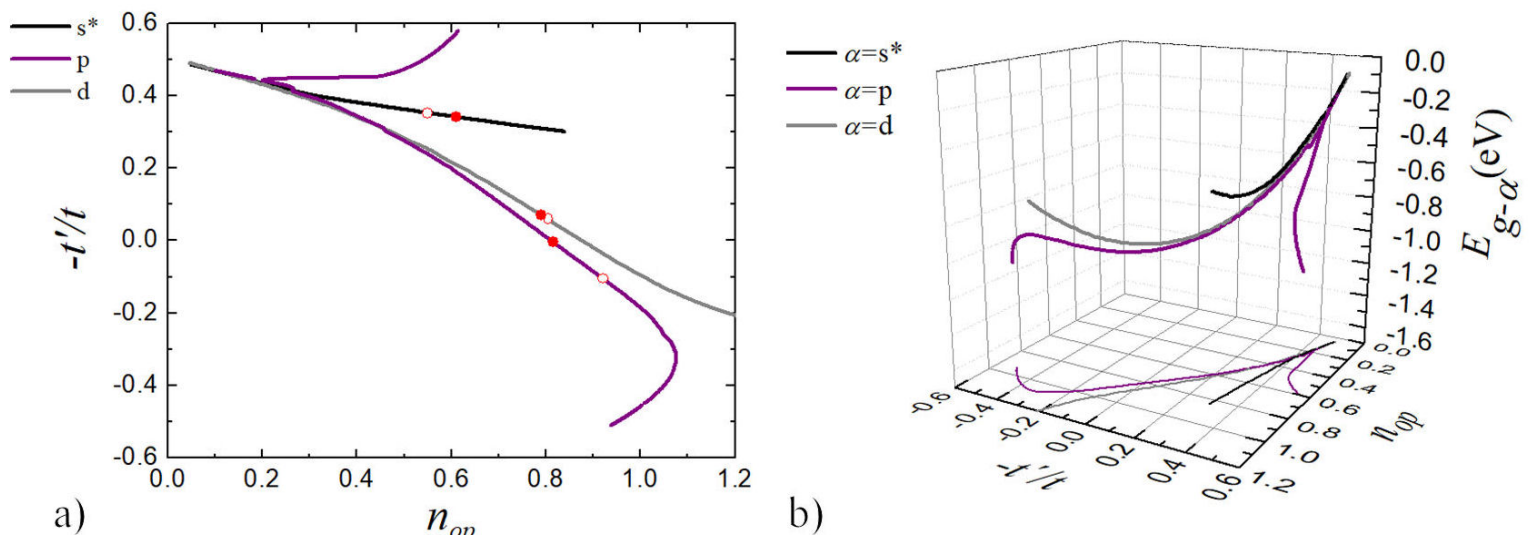

FIGURE 7. a) $\left(n_{o p},-t / t\right)$ states where the maximum critical temperature $\left(T_{c-\max }\right)$ is attained for $s^{*}-$ (black line), $p$ - (purple line), and $d$-wave (gray line) superconducting states with $\Delta t=0.5|t|$, and $\Delta t_{3}=0.05|t|$. The red open and solid circles correspond to minimum of $T_{c-\max }$, and ground state energy $\left(E_{g}\right)$, respectively. b) Ground state energies $\left(E_{g}\right)$ of the superconducting states depicted in Fig. 7a). 


\section{Conclusions}

For the first time, by means of the generalized Hubbard model, we have studied the variation of the maximum critical temperature $\left(T_{c-\max }\right)$, the gap amplitude $\left(\Delta_{p}\right)$ and the ground state energy $\left(E_{g}\right)$ of $p$-wave superconducting ground states in a square lattice within the $\left(n_{o p}, t^{\prime} / t\right)$ space for fixed values of the electron-electron interaction parameters, where $n_{o p}$ denotes the optimal electron density where the maximum $T_{c}$ is attained for a given value of the second-neighbor hopping $\left(t^{\prime}\right)$. In contrast to previous studies, here the effects of different values of $t^{\prime}$ on the superconducting properties were analyzed. The ground state energies $\left(E_{g}\right)$ were obtained for all optimal electron concentrations $\left(n_{o p}\right)$ where the critical temperature $\left(T_{c}\right)$ is maximum, for systems with given values of the second-neighbor hopping $\left(t^{\prime}\right)$ and $\delta_{3}$ and with $\Delta t=0.5 \mathrm{eV}, \delta=\Delta t_{3}=0.05 \mathrm{eV}$. It is important to emphasize that it is not possible to define a supremum value for the set of maximum critical temperatures $\left(T_{c-\max }\right)$, as occurs for $d$-wave superconducting states [9], where the minimum ground state energy is found for that state with the minimum value of $T_{c-\max }$. Moreover, the results presented in this work suggest that the $p$-wave superconducting ground states that reproduce $T_{c} \approx 1.5 \mathrm{~K}$, are found close to half filling $\left(n_{o p} \sim 1\right)$, and should posses $t^{\prime}<0$, in agreement with first-principles calculations. In addition, in this param- eter zone the Fermi surface is close and the single-particle excitation energy $\left[\Delta_{0}(\theta)\right]$ possess a clear $p$-wave pattern. Moreover, within this zone, the $p$-wave ground state energies are lower than the surrounding $d$ - and $s^{*}$-wave superconducting states. In contrast to previous works $[6,7,13]$, this study indicates that the Hamiltonian-parameter zone where stable $p$-wave superconductivity with low critical temperatures can be found embraces positive and negative values of $t^{\prime}$, and thus other superconducting materials whose pairing symmetry has not been established can be studied under the framework considered in this work. For example the iron-based superconductors, where the K-doping of $\mathrm{Sr}_{1-x} \mathrm{~K}-x \mathrm{Fe}-2 \mathrm{As}_{2}$, modifies the former lattice and electronic structure [17]. In addition, we can also investigate compounds such as $\mathrm{RuSr}_{2-x} \mathrm{Ba}_{x} \mathrm{GdCu}_{2} \mathrm{O}_{8}$ which contains both $\mathrm{Ru}-\mathrm{O}$ and $\mathrm{Cu}-\mathrm{O}$ planes, and could have superconducting gaps with different symmetries [18].

\section{Acknowledgments}

This work has been supported by UNACAR-2020 project, ENERXICO Project No. B-S-69926, and UNAM-PAPIIT IN109320. The authors thankfully acknowledge the computer resources provided by Laboratorio Nacional de Supercómputo del Sureste de México (LNS) and DGTICUNAM (project LANCAD-UNAM-DGTIC-180).
1. A. J. Leggett, A theoretical description of the new phases of liquid ${ }^{3} \mathrm{He}$, Rev. Mod. Phys. 47 (1975) 331, https://doi. org/10.1103/RevModPhys.47.331

2. Y. Maeno et al., Superconductivity in a layered perovskite without copper, Nature 372 (1994) 532, https://doi.org/ $10.1038 / 372532 \mathrm{a} 0$

3. T. Matsuzaki, N. Momono, M. Oda, and M. Ido, Electronic Specific Heat of $\mathrm{La}_{2-x} \mathrm{Sr}_{x} \mathrm{CuO}_{4}$ : Pseudogap Formation and Reduction of the Superconducting Condensation Energy, $J$. Phys. Soc. Jpn. 73 (2004) 2232, https://doi.org/10. $1143 /$ JPSJ.73.2232

4. M. Schmid, B. M. Andersen, A. P. Kampf, and P. J. Hirschfeld, $d$-Wave superconductivity as a catalyst for antiferromagnetism in underdoped cuprates, New J. Phys. 12 (2010) 053043, https://doi.org/10.1088/ 1367-2630/12/5/053043.

5. S. Ran et al., Nearly ferromagnetic spin-triplet superconductivity, Science 365 (2019) 684, https://doi.org/10. $1126 /$ science.aav8645

6. J. S. Millán, L. A. Pérez, and C. Wang, p-wave superconductivity in a twodimensional generalized Hubbard model, Phys. Lett. A 335 (2005) 505, https: / /doi.org/10.1016/j. physleta.2004.12.080

7. L. A. Pérez, J. S. Millán, and C. Wang, Spin singlet and triplet superconductivity induced by correlated hopping interactions, Int. J. Mod. Phys. B 24 (2010) 5229, https://doi.org/ $10.1142 / \mathrm{S} 0217979210057353$
8. J. S. Millán, I. R. Ortiz, L. A. Pérez, and C. Wang, FirstBrillouin-zone integration areas for anisotropic superconducting states, J. Phys. Conf. Ser. 490 (2014) 012221, https: //doi.org/10.1088/1742-6596/490/1/012221.

9. B. Millán, L. A. Pérez, and J. S. Millán, Optimal doping for $d$-wave superconducting ground states within the generalized Hubbard model, Rev. Mex. Fis. 64 (2018) 233, https: //doi.org/10.31349/RevMexFis.64.233

10. B. Millán, I. J. Hernández-Hernández, L. A. Pérez, and J. S. Millán, A comparison of optimal doping behaviors between $d$ - and $s^{*}$-wave superconducting ground states, Rev. Mex. Fis. 67 (2021) 312, https: //doi.org/10.31349/ RevMexFis.67.312

11. I. I. Mazin and D. J. Singh, Ferromagnetic Spin Fluctuation Induced Superconductivity in $\mathrm{Sr}_{2} \mathrm{RuO}_{4}$, Phys. Rev. Lett. 79 (1997) 733, https://doi.org/10.1103/ PhysRevLett.79.733.

12. R. Matzdorf et al., Ferromagnetism Stabilized by Lattice Distortion at the Surface of the $p$-Wave Superconductor $\mathrm{Sr}_{2} \mathrm{RuO}_{4}$, Science 289 (2000) 746, https://doi.org/10.1126/ science.289.5480.746

13. J. S. Millán, L. A. Pérez, and C. Wang, Chiral and non-chiral $p$-wave superconducting states from correlated hopping interactions, Phys. Status Solidi B 251 (2014) 2302, https : / / doi. org/10.1002/pssb.201451107 
14. L. A. Pérez, Ph.D. thesis, Superconductividad con simetría $d$ en el modelo de Hubbard, Universidad Nacional Autónoma de México, 2002, p. 66.

15. L. A. Pérez, O. Navarro, and C. Wang, Nonperturbative results for attractive Hubbard pairings in triangular lattices, Phys. Rev. B 53 (1996) 15389, https://doi.org/10.1103/ PhysRevB.53.15389

16. E. Dagotto, A. Nazarenko, and A. Moreo, Antiferromagnetic and van Hove Scenarios for the Cuprates: Taking the Best of
Both Worlds, Phys. Rev. Lett. 74 (1995) 310, https : / doi. org/10.1103/PhysRevLett.74.310

17. M. Pan et al., The Doping Effect on the Lattices and Electronic Structure in Superconducting Fe-based Compounds $\mathrm{Sr}_{1-x} \mathrm{~K}_{x} \mathrm{Fe}_{2} \mathrm{As}_{2}$, J. Supercond. Nov. Magn. 23 (2010) 985, https://doi.org/10.1007/s10948-010-0693-0.

18. L. T. Yang et al., Enhancement of superconducting transition temperature via $\mathrm{Ba}$ doping in $\mathrm{RuSr}_{2-x} \mathrm{Ba}_{x} \mathrm{GdCu}_{2} \mathrm{O}_{8}(x \leq$ 0.1), J. Appl. Phys. 95 (2004) 1942, https://doi.org/ $10.1063 / 1.1636829$ 